\title{
Early salivary changes in multiple myeloma patients undergoing autologous HSCT
}

\section{S.J.M. van Leeuwen', G.B. Proctor², C.M.J. Potting ${ }^{3}$, S. ten Hoopen', L.F.J. van Groningen ${ }^{3}$, E.M. Bronkhorst', N.M.A. Blijlevens ${ }^{3}$, M.C.D.N.J.M. Huysmans ${ }^{1}$ \\ 'Department of Dentistry, Radboudumc, Nijmegen, The Netherlands 2Division of Mucosal \& Salivary Biology, King's College London Dental Institute, London, UK ${ }^{3}$ Department of Hematology, Radboudumc, Nijmegen, The Netherlands Email: stephanie.vanleeuwen@radboudumc.nl}

\section{INTRODUCTION}

Oral mucositis (OM) is a common adverse event of conditioning therapy before a hematopoietic stem cell transplantation (HSCT). Saliva and especially salivary proteins play a major role in protecting the oral mucosa and changes may influcence the severity of $O M$.

\section{OBJECTIVE}

To examine early salivary changes in relation to OM in multiple myeloma patients treated with highdose melphalan (HDM) and autologous HSCT. Due to protocol changes at the hospital, the effect of cryotherapy was evaluated.

\section{METHODS}

Observational study in 2 parts

part A (2013) $n=12$ | part B (2015) $n=8$

Between parts $A$ and $B$ a standard protocol change including cryotherapy was introduced at the hospital. Study days

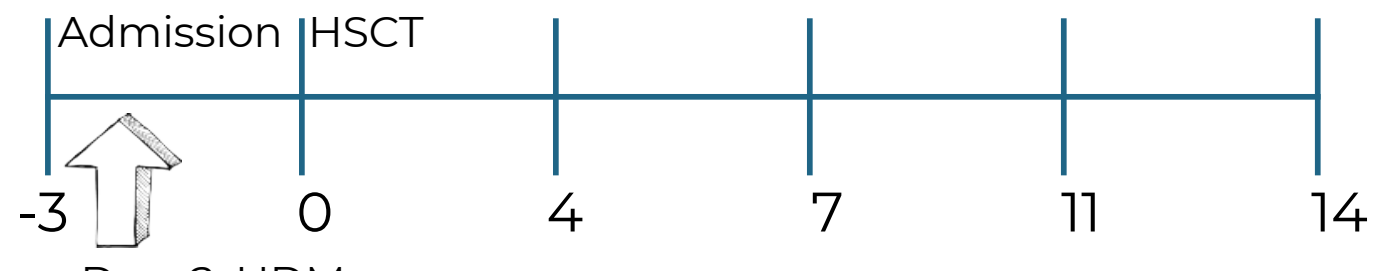

Day -2: HDM

- Collection of unstimulated and stimulated wholemouth saliva (UWS + SWS)

Scoring OM with OMNI (Oral Mucositis Nursing Instrument)
- Blood levels of polymorphonuclear leukocytes (PMNs) were obtained from medical charts

Laboratory analysis (only part B)

- Western blot: albumin

- ELISA: total IgA, lactoferrin + myeloperoxidase (MPO)

\section{Statistical analysis}

Scaled scores to correct for interindividual variation.

Paired t tests comparing days 0, 4, 7, 11 and 14 with day -3. Correlations calculated by applying meta-analytical techniques (inverse variance weighting) on individual correlations within patients over time.

\section{RESULTS}

- Trends of decreasing flow rates and Total IgA in UWS and SWS

- Positive correlation between OMNI scores and albumin and lactoferrin levels in SWS $\left(R^{2}=0.56\right.$, $\mathrm{p}=0.029$ and $\mathrm{R}^{2}=0.49, \mathrm{p}=0.043$, respectively)

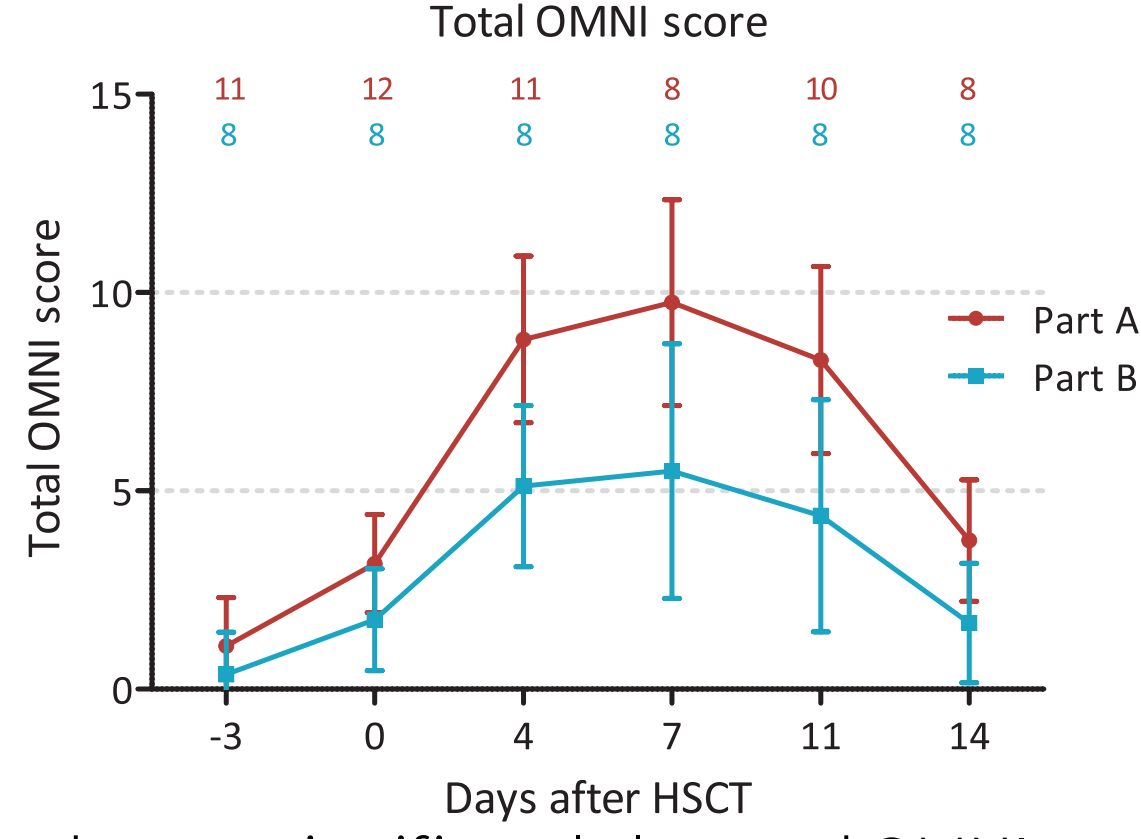

Cryotherapy significantly lowered OMNI scores in part B 95\% Cl [-3.87, -1.29], $\mathrm{p}<0.001$ (multilevel regression model)

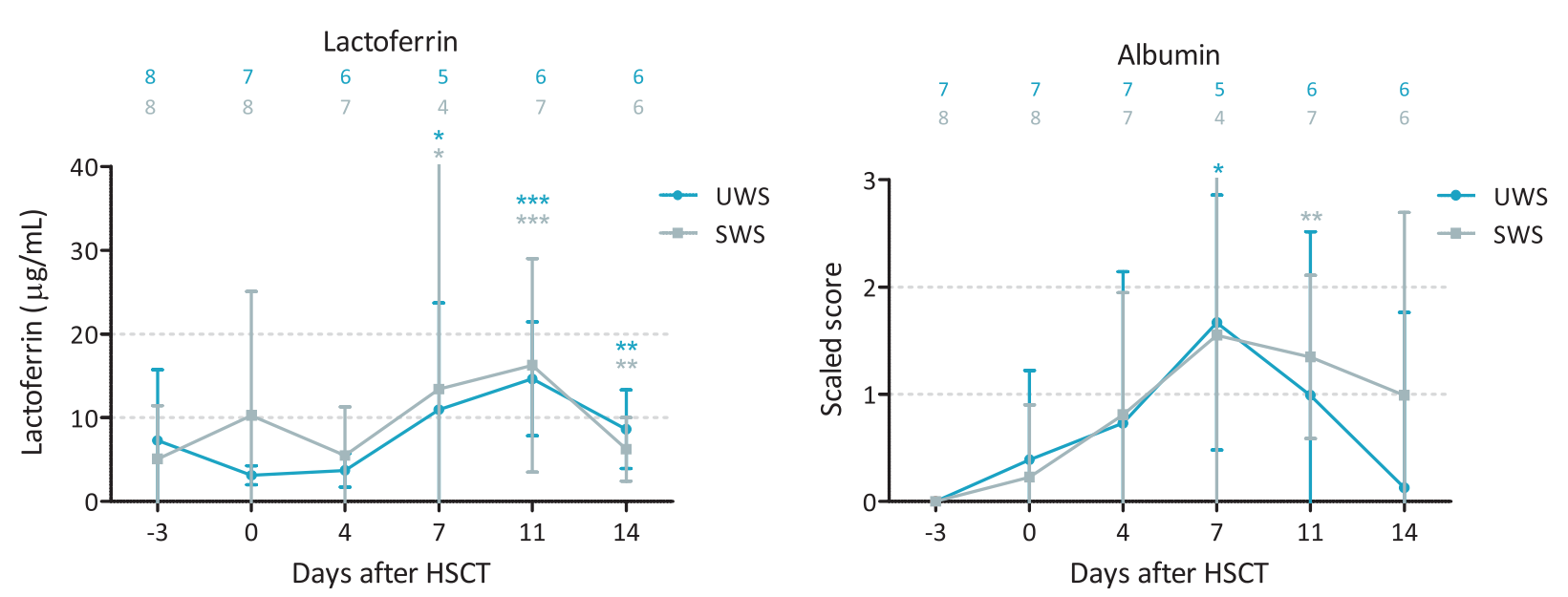

Increases in lactoferrin and albumin levels in UWS and SWS

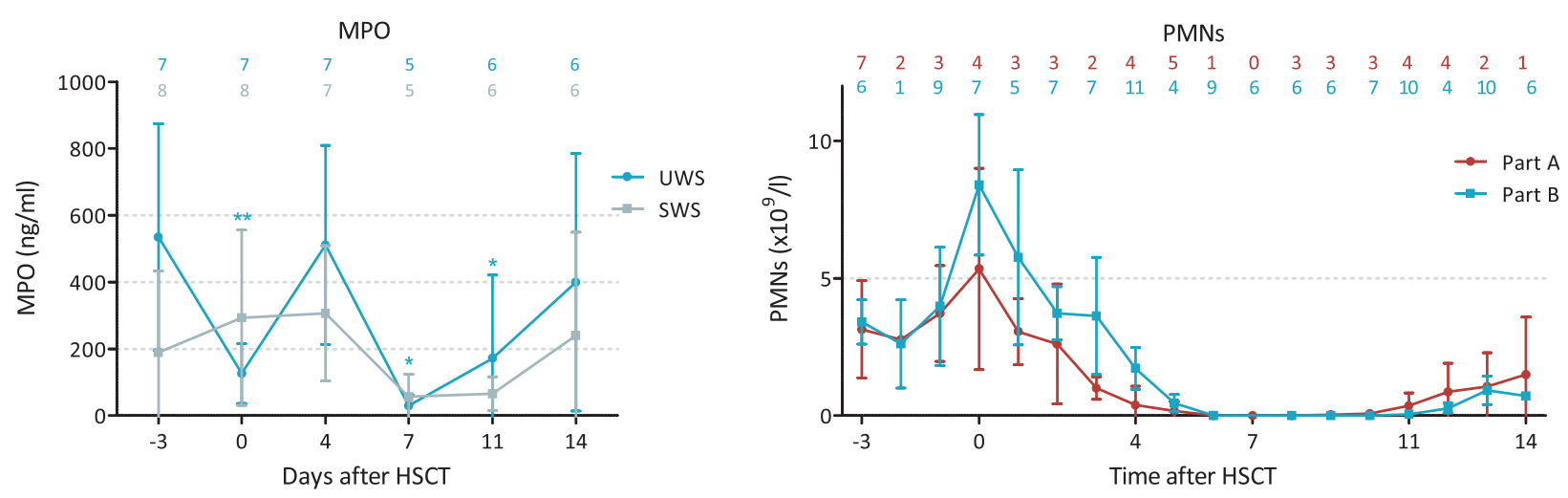

Fluctuating trend in salivary MPO levels reflect the changes in blood PMNs

\section{CONCLUSION}

